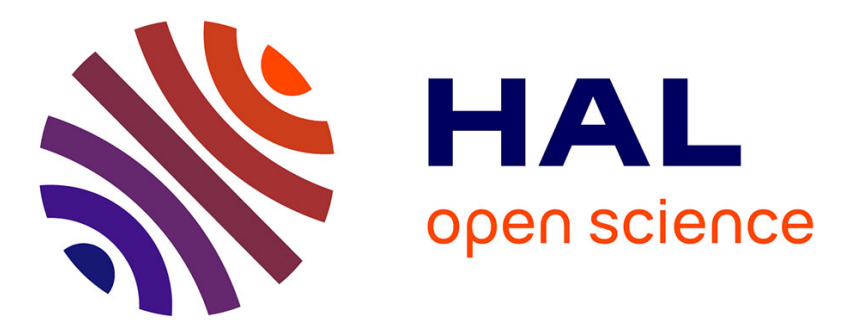

\title{
Enduits peints de Ribemont-sur-Ancre
}

Claudine Allag

\section{To cite this version:}

Claudine Allag. Enduits peints de Ribemont-sur-Ancre. Gallia - Fouilles et monuments archéologiques en France métropolitaine, 1982, 40 (1), pp.107-122. 10.3406/galia.1982.1855 . hal-01940309

\section{HAL Id: hal-01940309 \\ https://hal.science/hal-01940309}

Submitted on 27 Feb 2020

HAL is a multi-disciplinary open access archive for the deposit and dissemination of scientific research documents, whether they are published or not. The documents may come from teaching and research institutions in France or abroad, or from public or private research centers.
L'archive ouverte pluridisciplinaire HAL, est destinée au dépôt et à la diffusion de documents scientifiques de niveau recherche, publiés ou non, émanant des établissements d'enseignement et de recherche français ou étrangers, des laboratoires publics ou privés.

\section{(a)(1) $\$$}

Distributed under a Creative Commons Attribution - NonCommercial - NoDerivatives| 4.0 


\title{
ENDUITS PEINTS DE RIBEMONT-SUR-ANCRE
}

\author{
par Claudine ALLAG
}

Les enduits peints provenant du serteur des thermes ont été etudiés en aoùt 1980 au Centre deetude des peintures murales romaines a soissons. Ces thermes sont tres précisément datés : leur construction remonte aux premieres années du $\mathrm{II}^{\mathrm{e}}$ siècle ; leur destruction à la première moitié du siècle suivantº Les matériaux provenant de leur destruction étaient amassés dans une fosse voisine : mortier de sol, cendres, fragments d'enduit mural. Aucune trace d'incendie n'apparait sur les peintures; il n'y a donc pas eu incendie de l'édifice, mais bien démolition volontaire et entassement systématique des enrluits. Les cendres mèlées aux déblais proviennent sans conteste de la démolition des praefurnia et des hyporaustes, et nont aucun lien avec la destruction des peintures. La présence de tress grosses plaques de mortier $(40)$ a $50 \mathrm{~cm}$ de còté) confirme encore que le revètement pariétal a été volonlairement détaché et jeté - alors qu un abandon progresif donne une usure et un effritement des enduits caractéristiques.

Nous avons trié les fragments selon la nature de leur support épaisseur et composants des rouches de mortier), et nous avons tenté l'étude du décor à l'intérieur de chaque groupe. Ialleureusement, les éléments décorés sont trop peu nombreux at trop dispersés pour nous permettre une restitution, mème parcellaire, de la décoration, ce qui est d'autant plus regrettable que des peintures datées avec autant de précision sont rares, et que des décors reconstitués bien situés chronologiquement seraient précieux.

\section{GROIPE I.}

Les fragnents de re groupe onl presque tous quardé l'épaisseur fol ale du mortier, soil $80 \mathrm{~mm}$; le revers porte parfois l'empreinte des petits moellons de la construction. longs: de 15 c'm environ et hauls de $10 \mathrm{~cm}$.

Ce support consiste en (fig. 1 a) : une couche de preparation, posie directement sur le mur; elle est épaisse de (fi) a $70 \mathrm{~mm}$, d'une couleur blanc rosé. En plus de la chaux et du sable, elle contient de gros éléments de tuileau, des graviers, des coquilles. des brindilles dont les fibres restent bien

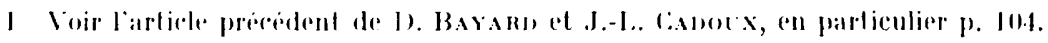



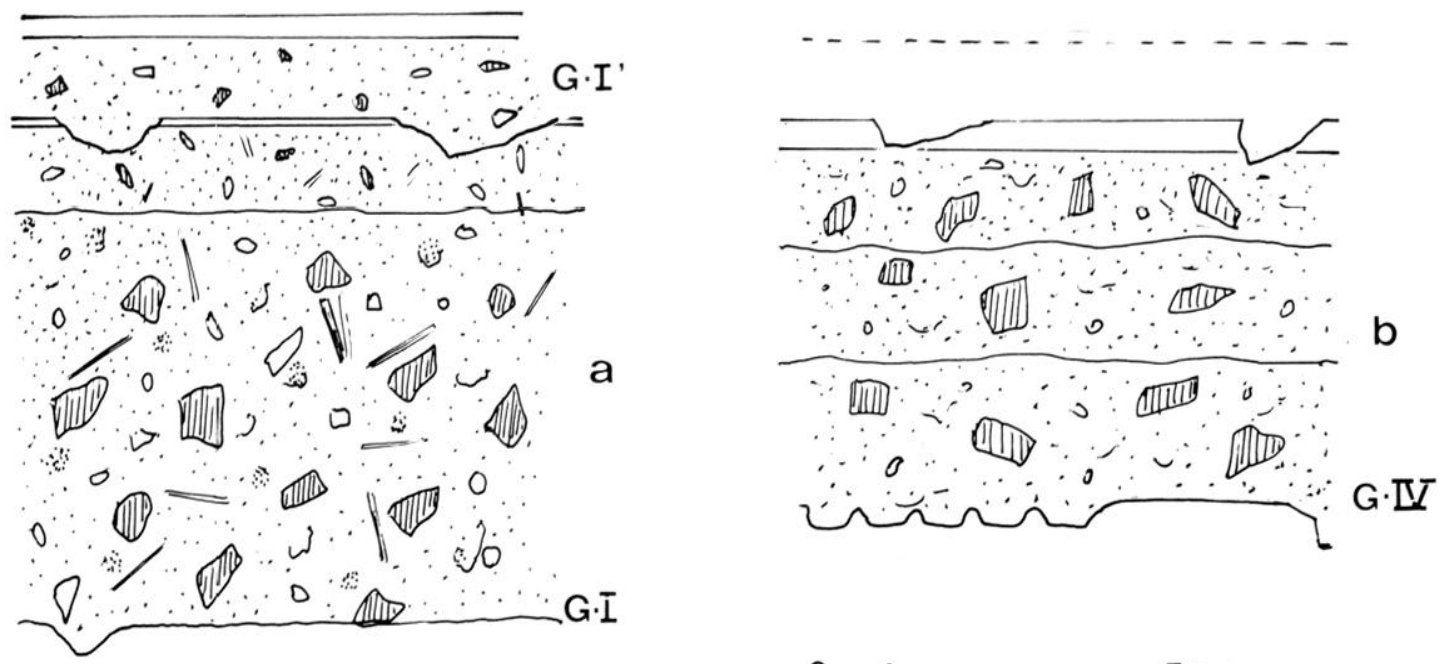

$0 \quad 5 \mathrm{~cm}$
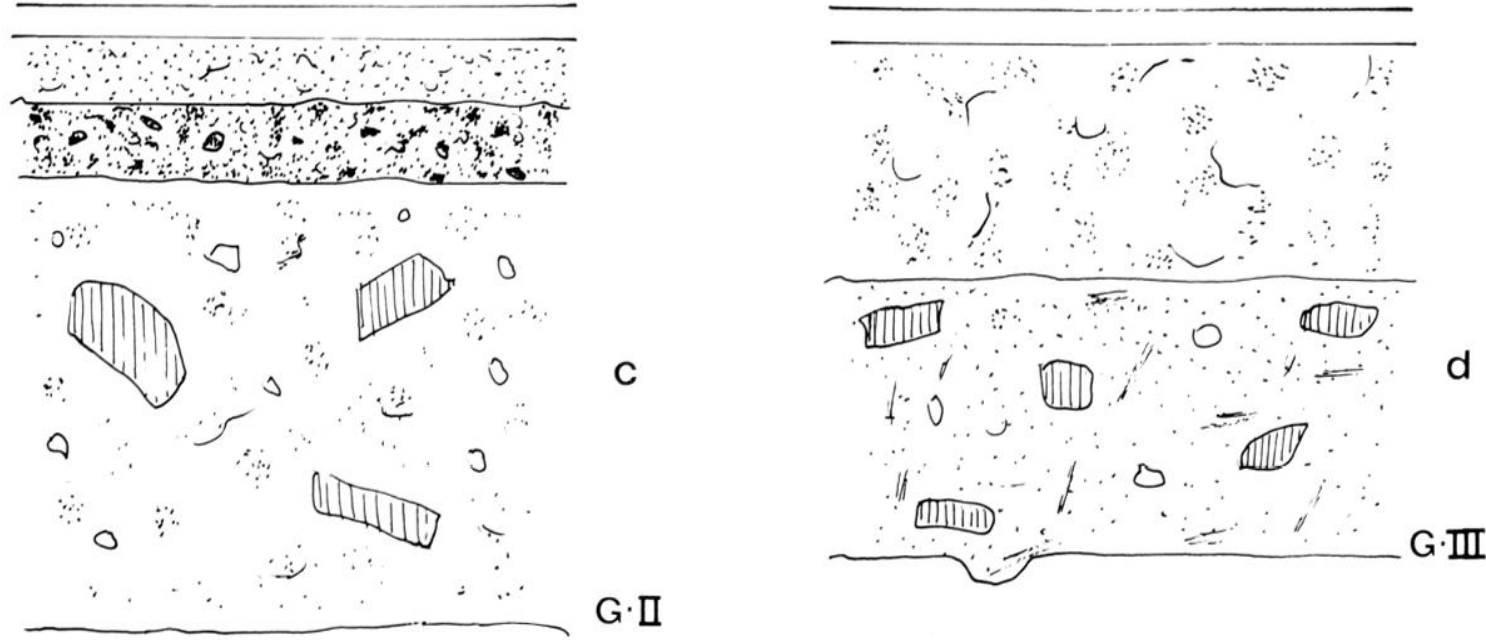

1 Coupe de's diffierents induits.

visibles. C'est un aggloméral très aéré, épais mais peu solide, caractérisé par sa consistance poreuse, friable et tris heterogrine (nodules de chaux, charge bien distincle); -.. une couche de transition, blanche, de $11 \mathrm{~mm}$ d'épaisseur en moyenne. Le mélange de chaux et de sable fin est allégé par un peu de paille hachée. Tuileau et graviers sont apparents, mais moins abondants que dans la couche précédente ; - la couche de surface, blanche $(1 \mathrm{~mm})$, est en chaux presque pure, régulière et bien lissée. Sia surface a été volontairement piquetée et rayée pour permettre l'adhérence d'une nouvelle couche de morlier, conservée sur certains echantillons, en vue de refaire le décor (groupe 1 bis).

la plupart des fragments appartenant a ce type sont blanc uni, re qui implique une composition triss simple.

Sur quelques morceaux, dont trois tris grosises plaques (K Th 12-2.2' el $2^{\prime \prime}$ ), apparait un motif de cercles sécanls alignes (lig. 2) peints en vert el soulignes de points (diam. des cercles: $120 \mathrm{~mm}$ ).

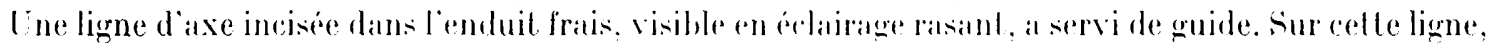

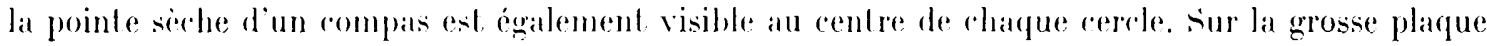




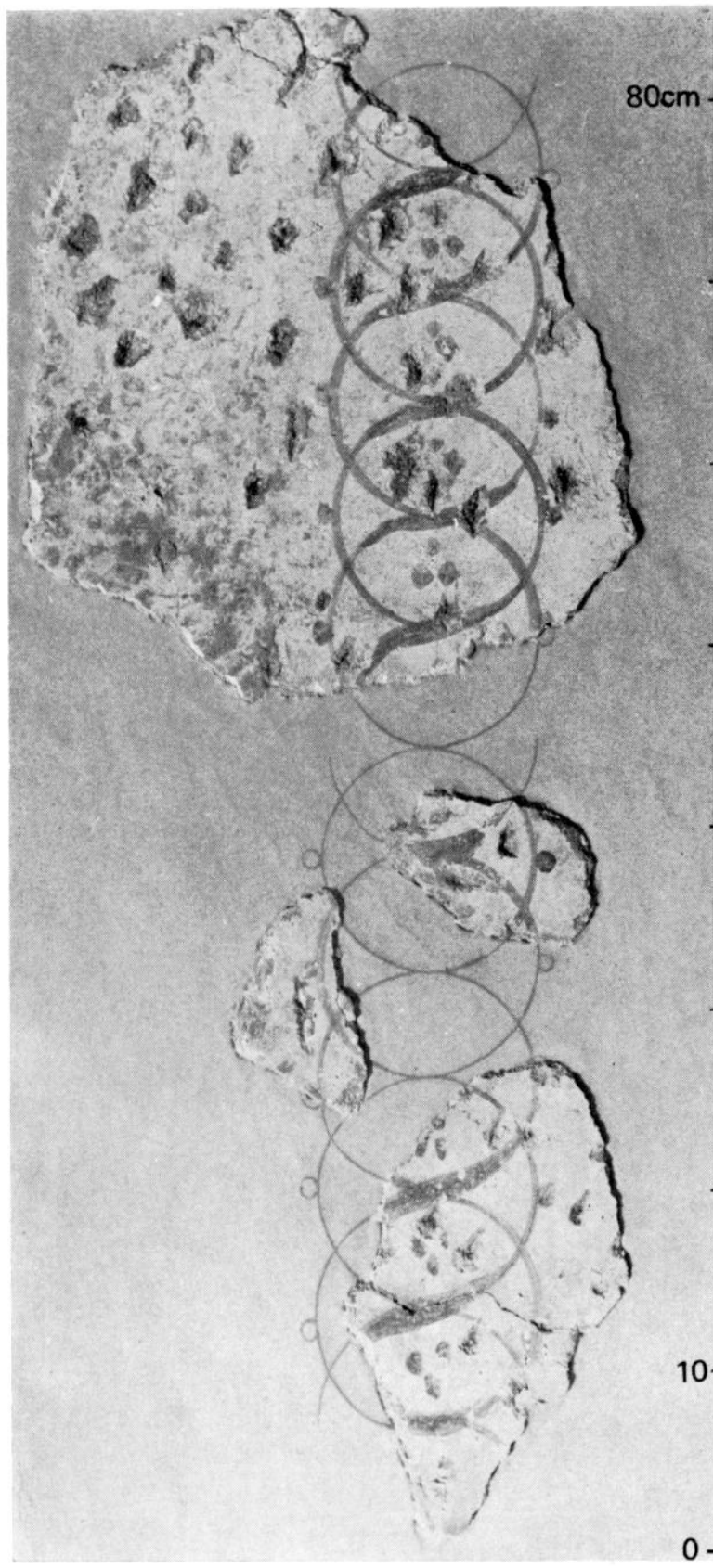

a

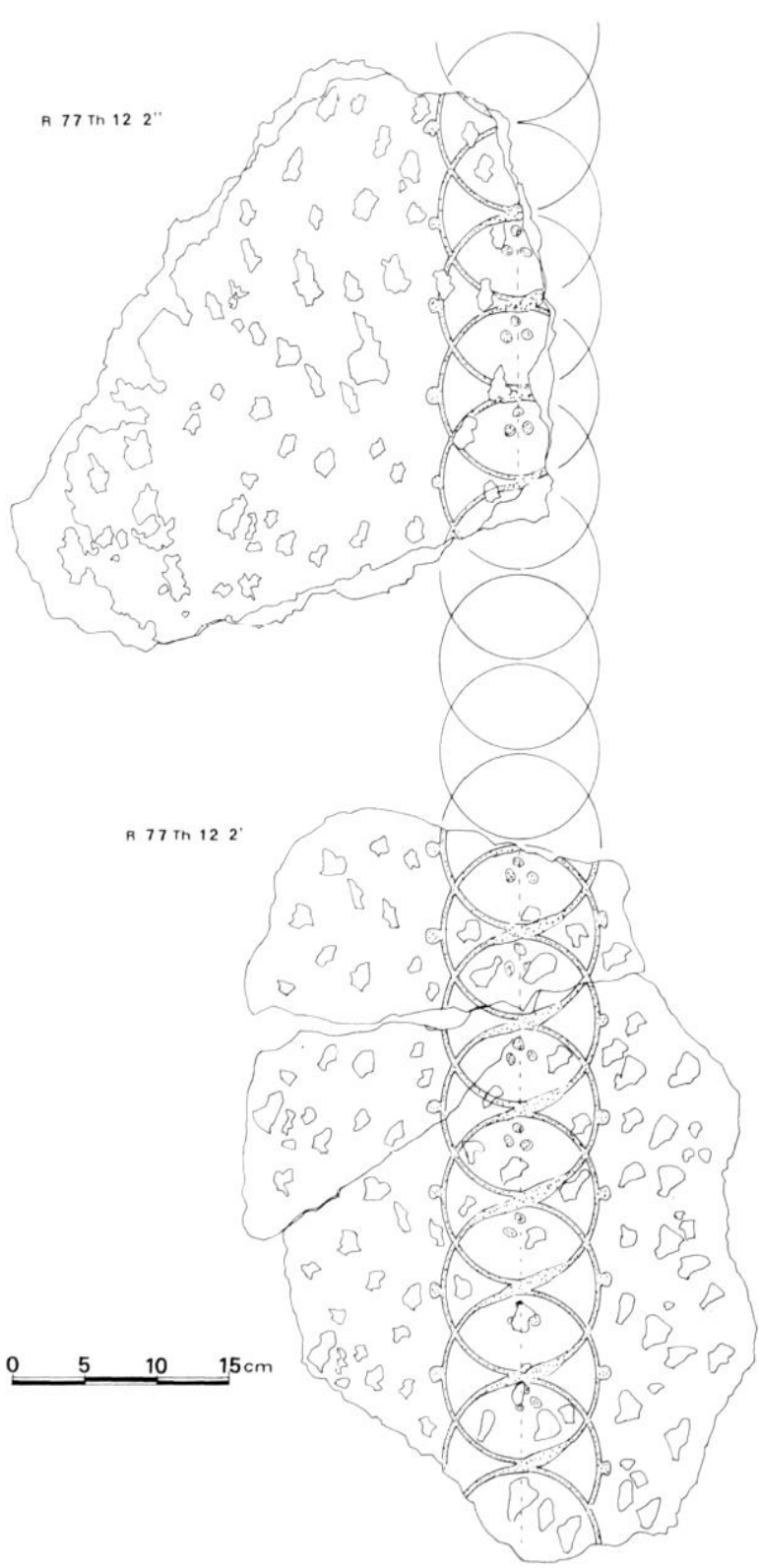

b

2 (iroupr I : ligne de cercles sur fond blanc: a, fragments mis sur support moderne, b, dessin des autres fragments.

(KT7 Th 1:2-2) la ligne de cercles doit ètre lue verticalement, grace aux empreintes des moellons du revers qui donnent indisculablement le sens des lits de pose.

Un cerlain nombre d’échantillons (dont la plaque 277 I 13-1) et une dizaine d'autres fragments présentent l'arète caractéristique de l'angle intéricur d'une pièce ; cette arête est bordée d'une bande

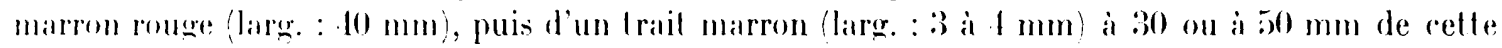
bande on distingue denx series . Exceplionnellenent, sur un fragment, la bande marquant l'arète est noirr. 


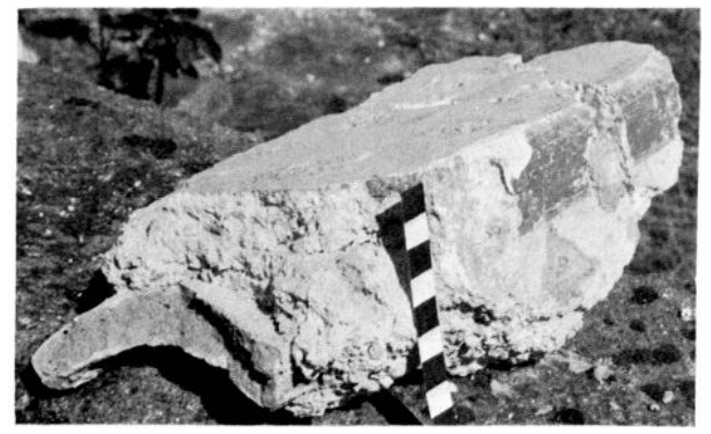

3 Groupe 1 : angle saillanl, sur imbrex, manque d'une hande marron rouge.

Plusieurs angrles droits saillants sont également soulignes par une bande marron rouge sur une lace, d'un trait sur l'autre ( $R$ 76-2). L'un d'entre eux présente une variante du support (figr. 3), due peut-être à sa situation dans la pièce, et qui ne l'exclut pas de ce groupe : une imbrex est incluse dans la couche de préparation. Sa couche de surface est aussi notablement plus épaisse (5 mm) (R 76 Th 12-1).

Lne série comporte un angle saillant en biseau (approximativement 1200). sans bande d'encadrement.

Enfin, deux fragments blances ont une surface non pas plane, mais concave.

Les angles marqués par les bandes colorés laissent supposer que l'encadrement général du décor était constitué de bordures marron rouge doublés de traits marron. les légères variantes dans leur lareeur ou leur écartement sont dues à la différence d'origine, à l’intérieur de la mème pièee, des divers échantillons qui nous sont parrenus. L'élément décoratif essentiel devait consister en ces lignes de rereles sécents découpant la surface du fond blanc des murs. Attesters vertiealement, olles pouvaient dessiner les quatre cotes de panneaux rectangulaires. Nous navons toutefois aucune rencontre entre ces lignes. Elles peuvent également créer un simple compartimentage vertical de la paroi.

Le principe d'un motif répétitif s'appuyant sur des lignes de cercles ou de demi-cercles, tangents ou sécants, est banal depuis le milieu du ger sièle, ot rle multiples borlures ajourées du quatrieme style pompéien sont ainsi concues. I)es lignes de cerceles tangents (par exemple a IIerculanum. maison de la (Ioison de Bois, ins. IIJ nos 11-12), ou des lignes de demicercles tangents et sécants (Herculanum, maison du Salon Noir, inv. IV, no 11 ) sont assez proches de notre dessin².

A Saintes, quelques enduits ont éte trowes en 1970 au chantier des Ateliers municipaux (fig. 4 a), que les conditions de fouilles un sauvetage diflicile - - nont pas permis de dater3. Ils proviennent des remblais sous lo sol inférieur diun édifice qui comporte deux etats surecssifs ; cela les silue done tres tót dams la chronologie relative, sans que l'on puisse fournir de date précise. Cies enduits présentent, comme à Ribemont, un décor

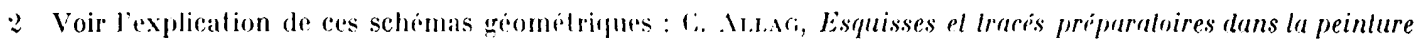

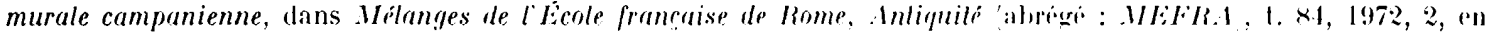

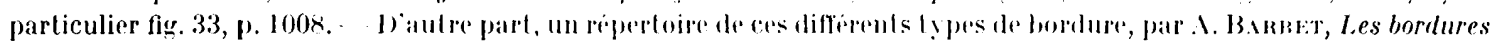

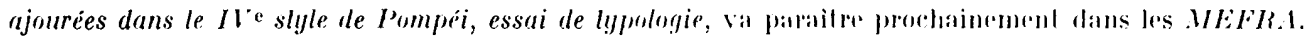

3 Ces enduits, dont j'ai eu commaissance grace a 11 . Rom reall of fi. Vienme, ne sonl pas publics. 

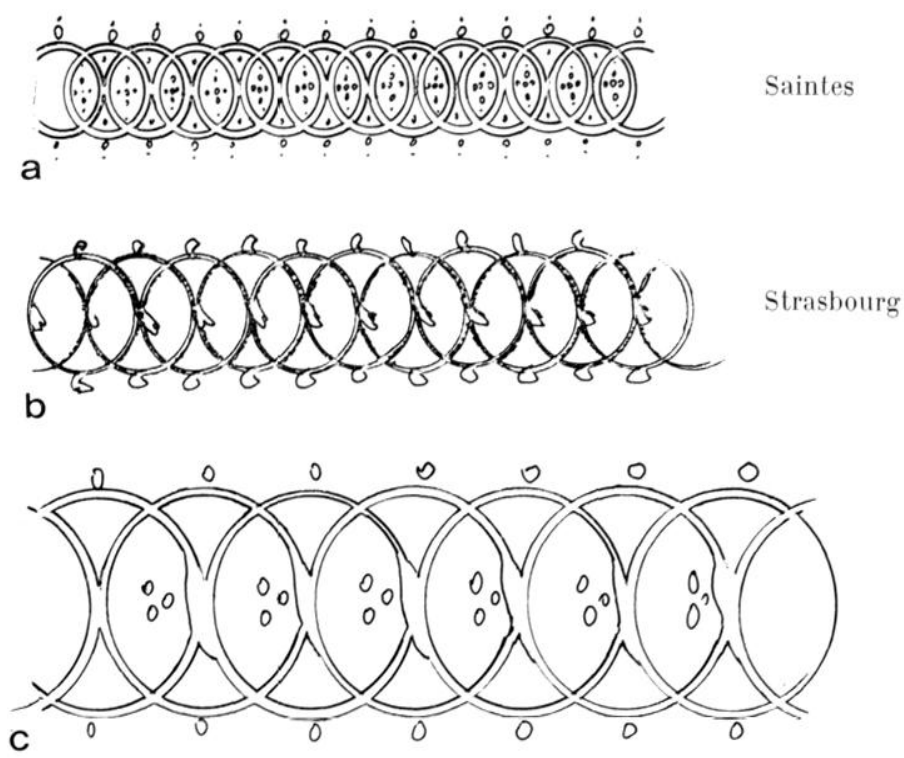

Ribemont

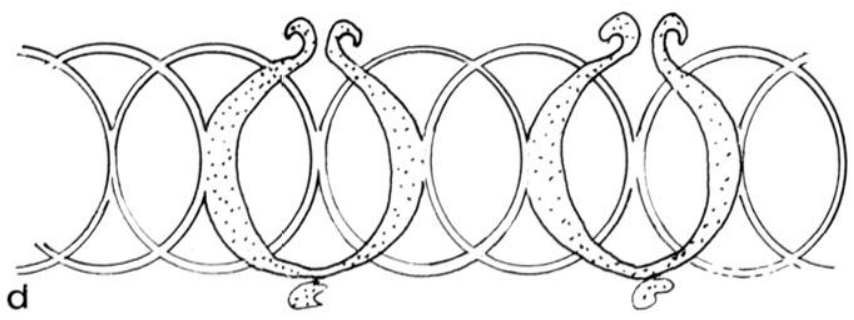

Betting

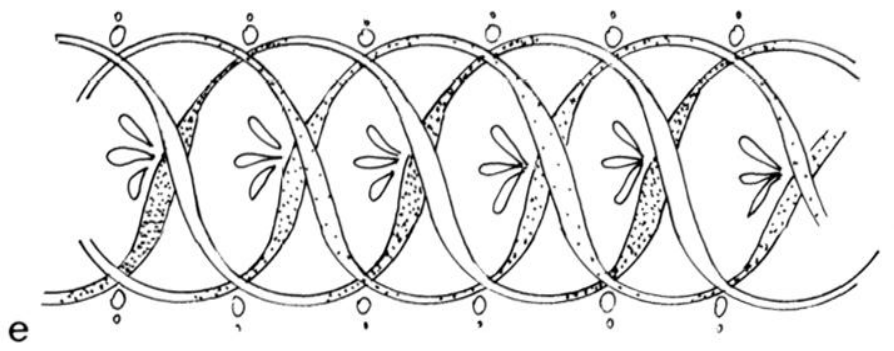

Nennig
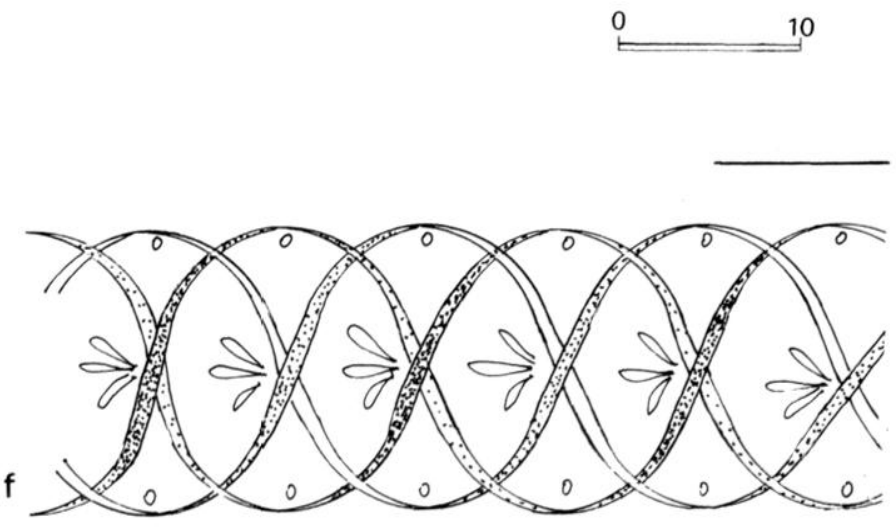

mosaĩque de la basilique Sainte-Constance à Rome

4 Bordures composenes de lignes de cercles tangenls at sicants. fi : rch. approximalive. 
constitué par la superposition de deux lignes de cercles sécants, peints en blanc sur un fond rouge, et soulignés de points sur les bordures extérieures et dans l'interville des ares de cercle. L'effet de transparence donné par ce motif peint en chair sur un fond foncé, et ses petites dimensions (5 $\mathrm{cm}$ de largeur au total) le font entrer dans la série des bordures campaniennes que nous venons de citer.

Une autre bordure de conception identique a été répertoriée à Strasbourg (fig. 4 b) ${ }^{4}$ : les fragments conservés témoignent d'un panneau rouge à figure féminine centrale, dont l'encadrement est constitué par les cercles sécants peints en blanc. Leur diamètre est de $6 \mathrm{~cm}$. Dans l'ensemble, ce décor, qualifié de "pompéien " par l'auteur de l'ourrage, ast. effectivement conforme à la tradition des peintures italiennes de la fin du zer siecle.

Le large dessin de Ribemont présente un aspect bien différent, et, si l'inspiration géométrique est la même, le résultat est très èloigné de ces encadrements assez fins. In motif aux dimensions rigoureusement identiques, aux coloris semblables (vert sur fond blane, avec cependant l'introduction du rouge), a èté trouvé en Jorraine, dans le frigidarium des thermes de la ville de Betting (fig. 4 d) $)^{5}$. Très proche aussi est celui de la villa de Nennig (fig. 4 e), près de Trèves (Allemagne) datée du me siècle. l)'un format très légrèrement supérieur $(14 \mathrm{~cm})$, il est peint en vert et beige sur un fond rouge. La restitution composée in silu, assez arbitraire, le fait figurer en deux frises parallèles au-dessus et aul-rlessous de la plinthe. Les fragments réels, non replacés dans leur contextr, sont présentés au musée de 'Trèves.

Malgré l'épaississement des brins aux entrecroisements, le décor de Ribemont-surAncre, ainsi que celui de la villa de Betting, reste assez proche du schéma purement géométrique. A Yening au contraire, lutilisation de couleurs alternées en fait des rubans entrecroisés. C'est sur une trame géométrique analogue qu'est hase le motif traite en mosaïque, répertorié comme "quadruple ruban ondé " ${ }^{7}$. Nous donnons comme comparaison la bordure sur fond blanc d'une mosaïque de voùte (fig. 4 f) a la basilique Sainte-('onst ance à Rome (rve siècle). Les palmettes intermédiaires, semblables sur la mosaïque ot sur la peinture de Nennig, sont peut-être esquissées a Ribemont sous la forme des Irois points verts, ce qui voudrait dire que l'inspiration est la mème, mais rendue de facon beaucoup plus schématique. On remarquera également que l'emploi de la couleur verte seule ot le renflement des lignes donnent au motif un aspect plutòt végétal, et l'éloignent, autant que l'agrandissement du format, des bordures d'encadrement originelles pour le rapprocher de certains rinceaux en volutes utilisés en frise. Le mélange de ces tendances est curieux.

Notons qu'une version plus simple de ces rubans ondés - simple entrelac's de deux brins -, bâtie sur une seule ligne de cercles tangents, se trouve également an peinture of en mosaïque : à Andilly-en-Bassigny, la bordure du plafond d'une villa, dont la const ruction

4 R. FonkER, Strasbourg-4rgentorale, Strasbourg, 19:2, 1. II, chap. 7, p. 12:2-4:9.

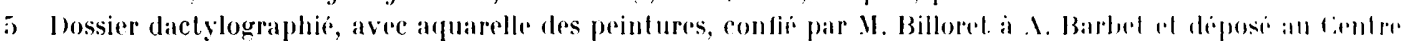
dielude des peintures murales romaines.

6 Trèves, musée, no inv. 00381193 c.

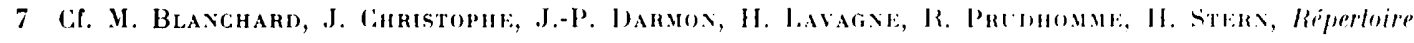
graphique du décor géomélrique alans la mosaique antique, dins Bull. de l Issociation Internationale pour l'ilnule de la Mosaique Antique, 4e fasc., mai 1973, 1. 47, n" $2: 25$. 
remonte au début du n ${ }^{\mathrm{e}}$ sièle, est constitué par deux brins, rose ch vert pour l'un, rouge el vert pour l'autre, se croisant autour d'une guirlande rigide qui suit la ligne d'axe du dessine. Les comparaisons données - - une peinture du musée du Bardo, sans guirlande, mais avec des fleurons au centre de chaque cercle, et la mosaïque dionysiaque de I)jemila, où l'articulation du motif, bien que plus enrubanné et plus fleuri, est la même -- attestent bien que pour cette série de décor, le répertoire est facilement interchangeable entre la peinture et la mosaïque.

Le motif de Ribemont peut constituer, avec les quelques exemples cités, l'amorce d'une série, apparaissant dans les provinces du Nord-Est à partir du I $^{\mathrm{e}}$ siècle : il s'agit du développement d'un motif né des bordures géométriques, mais beaucoup plus proche, par le traitement et les dimensions, des dessins de ruban ondé plus répandus ou du moins mieux connus en mosaïque. Des trouvailles ultérieures apporteront, espérons-le, des précisions.

L'ensemble des fragments de ce groupe suscite quelques remarques : le nombre des angles rentrants ou saillants est exceptionnellement grand par rapport is la surface de revêtement conservè ;

les blocs en angle droit saillant conviennent moins it une embrasure de porte ou de fenètre, où l'ouverture est souvent oblique, qu'à un décrochement sur la paroi. Én revanche, les angles en biseau correspondent a la première altribution;

la grande échelle du motif décoratif convient bien à une pièce asse\% vaste, d'autant plus que le nombre important de gros fragments sans décor, et l'absence de lignes perpendiculaires sur les fragments de bordure conservés impliquent des champs unis de surface considérable ;

étant donné leur forte concavité, trop marquée pour convenir à une voûte, que rien par ailleurs dans l'architecture ne permet de supposer, les fragments courbes doivent plutòt provenir d'une petite niche creusée dans l'épaisseur d'un mur. La présence d'une ou de plusieurs niches n'aurait rien de surprenant - c'est un aménagement intérieur habituel dans les bains.

D'après le plan des thermes, la pièce à laquelle conviendrait le mieux ce décor est sans doute le caldarium : c'est en effet la plus vaste, celle où les éléments décoratifs de grande dimension sont le plus à leur place ; c'est la seule où de multiples décrochements (six angles rentrants et surtout les deux refends) peurent justifier les angles de l'enduit. Linfin, rappelons qu'une réfection dont on ignore l'envergure, mais qui a touché de façon certaine le praefurnium du caldarium, a pu morlifier 'e secteur. On aimerait donc pouvoir attribuer à la même phase de remaniement la rénovation du décor peint, mentionnée comme couche 1 bis, après piquetage de la surface.

\section{GROUPE IV.}

Ce groupe, enregistré et numéroté d'abord comme groupe indépendant. est présenté à la suite du groupe I en raison d'une série d'analogies qui nous sont apparue's à l'étude.

Le support (5) mm au total) est constilué de trois couches de mortier de tuileau (fig. 1 h), toutes trois de composition et d'épaisseur équivalentes : chaux, sable, et tuile pilée en gros fragments

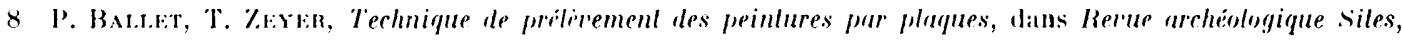
1981, p. $12-36$. 


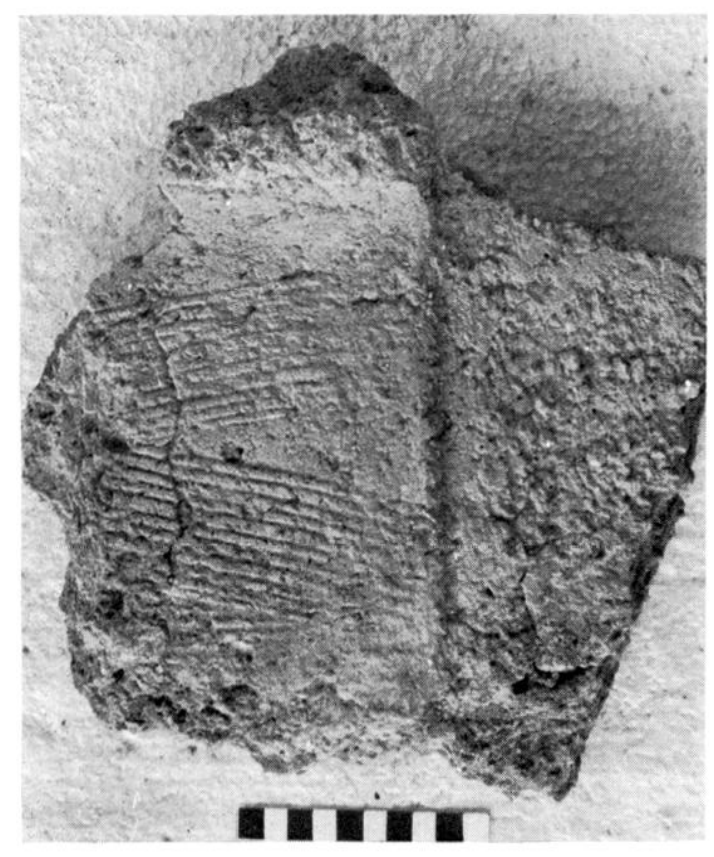

¿ Compe IV : revers de l'enduit, avec empreintes de lubulus.

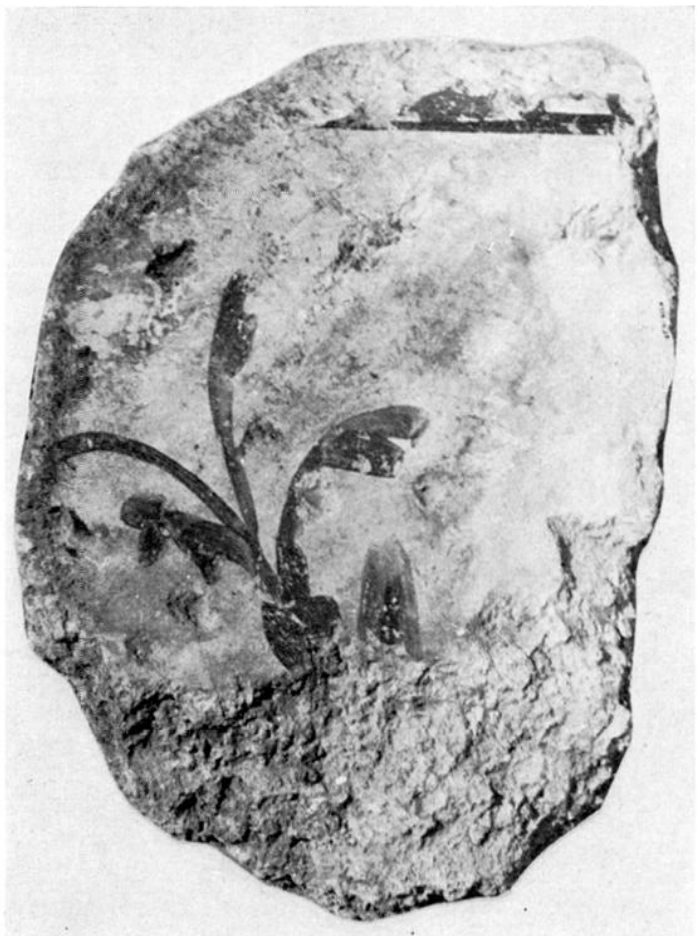

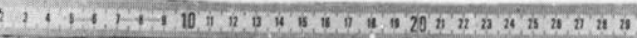

6 Groupe IV : feuillages sur fond blane.

distincts. Le revers des fragments porte l'empreinte de lubuli stries (figr. 5), ì rebords arrondis; la couche de surface est blanche, épaisse de $3 \mathrm{~mm}$, faite de chaux et de sable lamisé tris fin. Eille présente les mèmes traces de piquetage que celle du crroupe I.

En plus de quelques fragments blanc uni, ce groupe se limite à quatre plaques a feuillages jaune vert sur fond blane ( $\mathrm{K}$ Th 1320 et 20$)^{\prime}, 21$ el $21^{\prime}$ ) (fig. 6i, dont l'une porte l'amore d'une bande de bordure ou de séparation a motifs géométriques rouge et noir (K Th 1:321).

Le support en mortier de tuileau est bien particulier. et distinct du groupe précédent. Pourtant, l'aspect général de la surface, avec les fonds blancs soigneusement lissés et polis, lui ressemble beaucoup. De plus, l'enduit présente les mèmes piquetages, et les vestiges d'un mortier de réfection identiques à ce que nous arons vu. Yous sommes donr peut-ètre en présence des vestiges d'une seconde pièce. contemporaine de la première, et dont le décor aurait été rénové en mème temps. Nais les fragments sont si rares, el le décor representé si limité, que nous penserions plutòt à une zone particulière de la méme pièce : il peut s'agir par exemple de la partic basse du revètement, si le mortier a été étendu, romme il est fréquent. en zones horizontales sucessives, avec eventurllement de légeres variantes dans leur composition. In motif végétal conviendrait hien, d'autant plus quiaucun fragrment, dans le groupe 1, ne peut itre attribué à une plinthe. On a pu vouloir renforcer la partie inférieure des murs par une triple couche de mortier de tuileau. Ces fragments peuvent aussi provenir d'un secteur où le décor différait de celui du reste de la pièce, et où un support particulièrement solide était nécessaire : pourtour de bassin par exemple. 


\section{(AROLPE I bis.}

liette appellation recourre le deuxième état du décor $I$.

La réfection du décor n'a consisté qu'en une superposition de couches; après piquetage de la surface peinte précédente, on a passé une seule couche préliminaire de $10 \mathrm{~mm}$ (fig. 1 a), légèrement rosée, en chaux chargée de sable, de graviers et de petits éléments de luileau dont le calibre n'excède pas 2 a $3 \mathrm{~mm}$. la couche de surface, de 2 ou $3 \mathrm{~mm}$, est en chaux presque pure, tres poreuse, friable, et très abîmée.

Les fonds blanc uni recourraient les parties précédemment décorées ; en particulier, nous avons détaché celle couche sans décor sur la grosse plaque $\mathrm{R} 77$ Th 122 pour retrouver la ligne de cercles sécants. En revanche, des champs vert clair à lignne vert foncé recouvraient des zones blanches : la largeur de l'ensemble est indéterminée, au moins égale à $120 \mathrm{~mm}$. Les bandes de bordure marron rouge ont été repeintes à peu prìs identiques.

sur le fragment concave, que nous avons interprélé comme un revètement de niche, et qui était primitivement sans décor, a été peint un fleuron noir, aux pétales bleus, encadré de rouge.

sur les autres frargments, l'altération de la couche picturale est telle que l'on ne décèle parfois que des traces d'un champ brun ou violet, sans pouvoir reconnaitre la moindre forme.

\section{GROUPE III.}

Couche de préparation (fig. 1 c) : épaisse $(60 \mathrm{~mm}$ env.), mais aérée et trés hétérogène, elle comporte une proportion importante de chaux, avec de gros morceaux de tuileau et des graviers. $\Lambda u$ revers, quelques fragments présentent les empreintes caractéristiques des rebords de legulae;

- couches de transition : on distingue deux couches, de 7 a $8 \mathrm{~mm}$ chacune. la première posée est colorée en rose franc par la brique pilée et bien mélangée à la chaux et au sable. La seconde est. beige rosé, moins chargée en tuileau; couche de surface : blanche, de 3 ou $4 \mathrm{~mm}$ d'épaisseur, elle est à peine chargée en sable trìs fin.

Cous avons pu reconnaitre successivement : un ensemble homogìne avec une bande rouge bordée de brun d'un còté, de gris de l'aulre (R 77 I 13 22). Sur certains fragments, des traits obliques, gris également, relient la bordure grise à une autre bande identique (R 77 I 1324 ).

La bande rouge doit ètre vue horizontalement et interprétée comme un entablement, dont la bande grise est l'ombre portée (fig. 7). La ligne parallèle grise et les traits obliques figurent un plafond a caissons, vu sous l'entablement. Les fragments conservés donnent deux lignes de fuite différentes : nous avons donc ou bien un seul panneau avec vue axiale et perspectives symétriques convergentes, ou bien deux panneaux placés latéralement sur la paroi. Les panneaux blancs sont encadrés de filets gris, près desquels apparaissent parfois des pans de rubans rouges;

un fond blanc avec deux filets marron parallèles;

un champ vert clair, bordé d'une bande bleue, longeant un fond blanc;

divers fragments a fond vert, certains avec un filet rectiligne vert foncé, d'autres avec des bandes brunes ou vert foncé peu lisibles.

Le décor d'origine architecturale (entablement et caissons) correspond à l'un des poncifs les plus utilisés de la peinture romaine où des constructions légères à colonnettes et entablement en perspective constituent l'élément essentiel de cloisonnement de la surface. Les parois peintes sont traditionnellement divisées en trois par un édicule central délimitant un panneau axial et deux latéraux symétriques. Parmi les multiples exemples italiens où ce type de représentation constitue un classique, nous en mentionnerons un seul, celui de la maison "aux deux alria " à Herculanum (fig. 8), a fin de mieux saisir l'effet d'ensemble du décor dont proviennent les fragments de Ribemont, indépendamment d'une richesse 

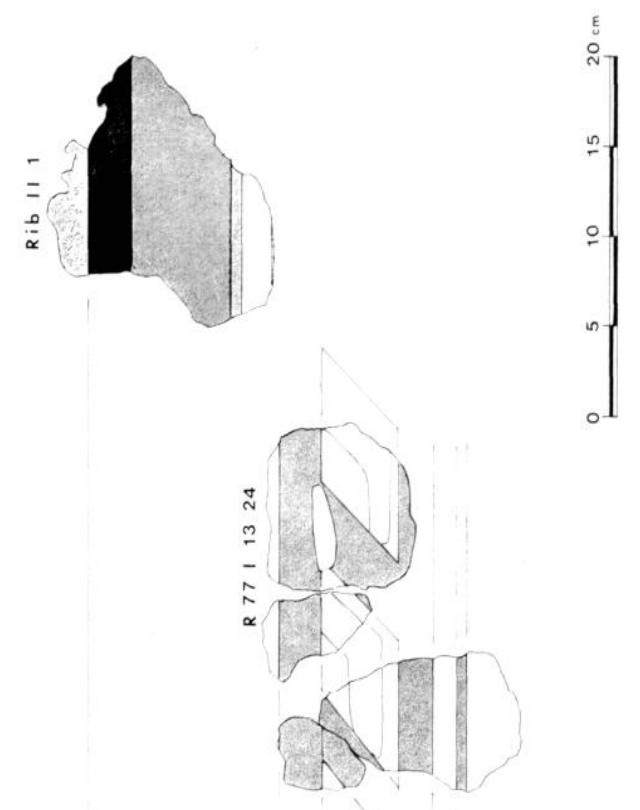

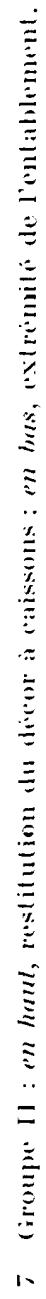
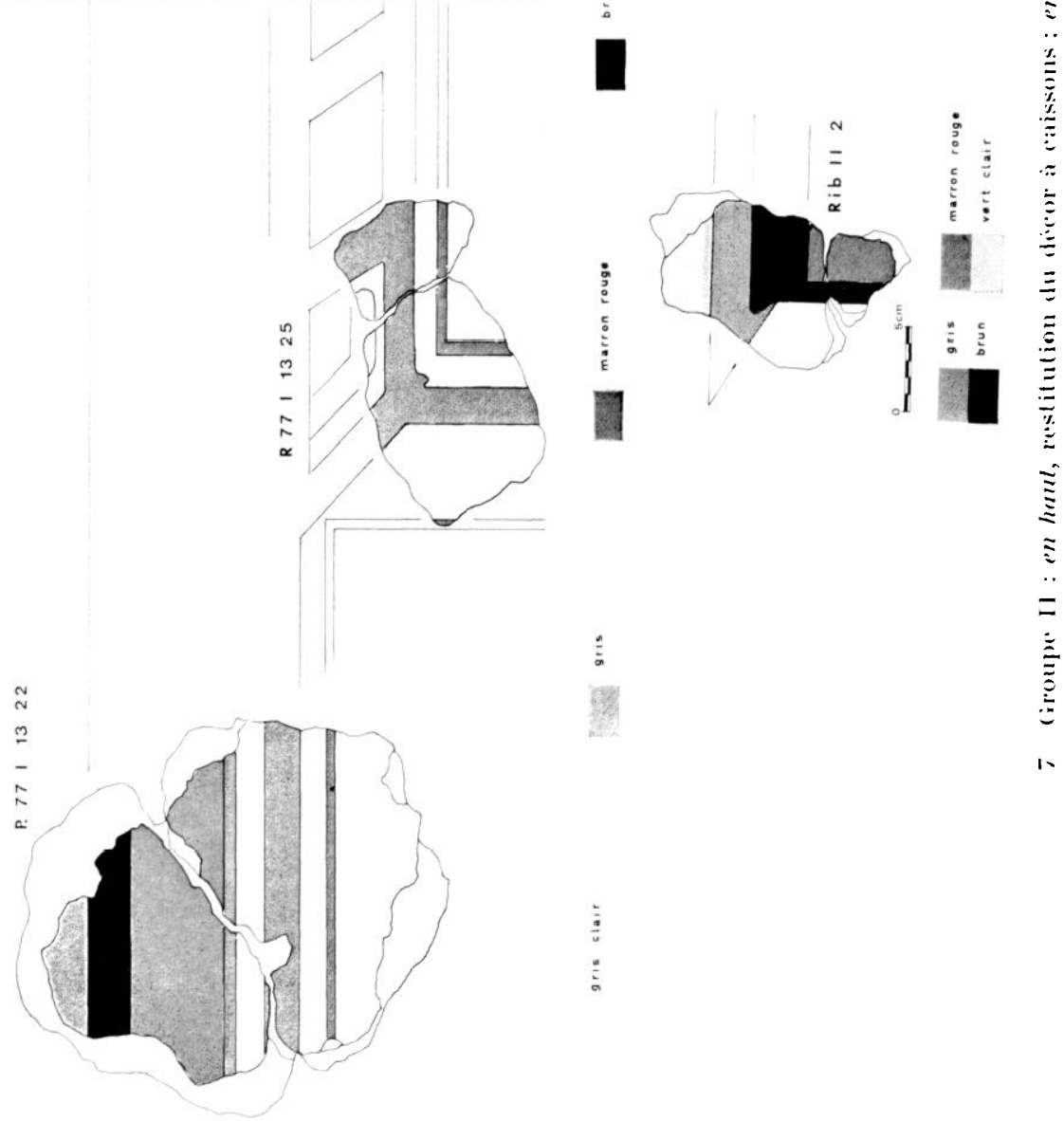

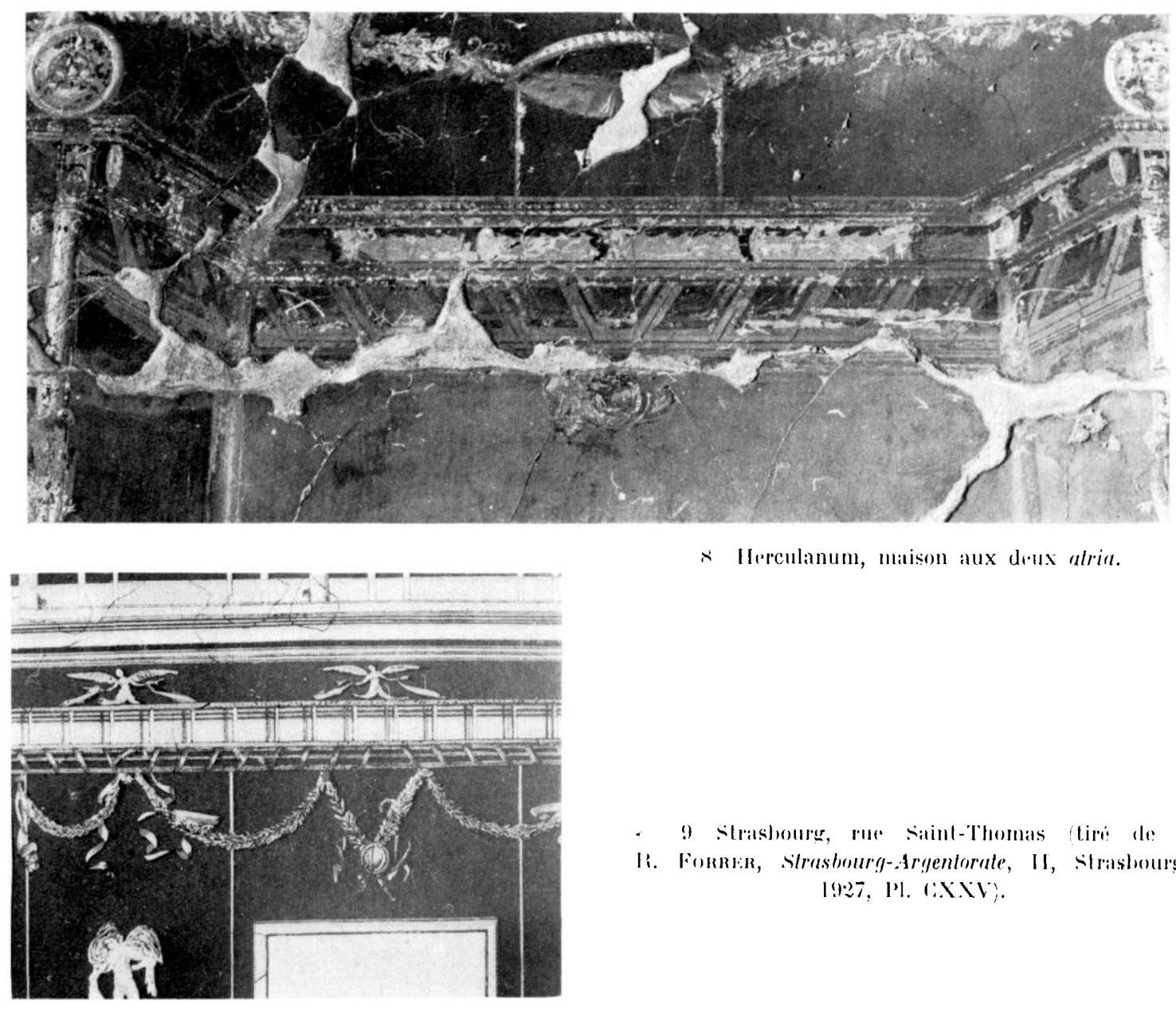

* Hereulanum, maison aux dertix atrit.

9) Strasbourer, rue satul-Thomas tiri de: R. FonRin, strasbourg-Argentorate, II, Slrashourg, 1927, P. (:XXV).

de:orative lien éloignée de la simplicité de la paroi grallo-romaine. Ilans les provinees, ce sist eme est nettement moins utilise. Il n est toutefois pas inconnu. Ainsi a Ilolstein (canton de Bàle) quelques fragments ont permis de reconnaitre un dessin de plafond a caissons peints en ocre. Yais il est situé en eouronnement à une suecession de panneaux en "laux marbre" (opus sectile), ce qui l'éloigne de son origine architecturale. La décoration du frigidarium des thermes de la villa dont ils proviennent est datée du milien du $\mathrm{uJ}^{\mathrm{e}}$ siècle?. A Trèves également, un décor de la Gilberlstrassero témoigne d'une colonne schématique et de caissons en perspective limitant un panneau blane; le traitement en un ramaïeu de blew gris ronne un effet assez proche de celui de Ribemont.

Uentionnons encore la peinture d'un long corridor d'une maison du II $^{\mathrm{e}}$ siecle à Leicester (Crandr-lBretagne), où les larges panneaux sont surmontés d'entablements à dessins de caissons. Chaque panneau a un axe central avere perspective convergente. Mais les architectures plus compliquées qui sönterealent entre eux, et l’intégration de guirlandes

9 B. Kapossy, Rümische Wundmalereien ans .Munsingen und IIolstein, dans delu Bernensia, IV, Berne, 1966. (en particulier p. 34 a 36 et pl. 11.

11) P. Sturen, Rijmische Wandmalerpie in Trier, dans Trierer Zeitschrift, 3x, 1949, p. 5)-fix. 
fleuries et de figures humaines apportent ì l'ensemble une richesse et une complexitó que nous n'arons pas à Ribemont ${ }^{11}$.

En Ciaule, ce motif n'est pas non plus très courant. si la division de la surface par des colonnettes et des entablements est maintenue, ces éléments architecturaux sont extrèmement simplifiés, et sourent réduits à de simples bandes plates sans aucun modelé. A Strasbourg, la restitution donnée par R. Forrer (fig. (9)12 montre des caissons très schématiques, copiés sans doute sur un modèle mal compris par le peintre et réduits à des traits obliques sans efet de profondeur. Au-dessus, une étroite balustrade porte des monstres ailés. Au-dessous, des panneaux à guirlandes sont rythmes par de simples traits vertiraux rn guise de colonnes portantes.

Par ailleurs, nous avons connaissance d'une peinture provenant de Chartres ${ }^{13}$, ou des caissons sont représentés sous l'entablement. In décor de Narbonne, représentant un édicule en perspective soigneusement peint et bien conservé, constitue, en Ciaule. une exception ${ }^{14}$. L'architecture peinte de Ribemont offre done l'intérêt de comporter, malgré la schématisation extrême des supports verticaux en simples lignes grises, la vue d'un plafond en perspective. Peut-être cependant la rareté des exemples analogues en Ciaule vient-elle surtout de la difficulté d'interpretation de trop de fragments isolés, où les lignes obliques sont difficilement identifiables.

La gamme des couleurs employees (beaucoup de marrons et de verts), la bande vert elair traversée par une ligne vert sombre, la mauvaise qualité de la couche de surface, fortement érodée et très pulvérulente, sont très semblables a celles que nous avions noté sur le deuxieme état du groupe précédent (groupe I bis). Faut-il en conclure à une contemperanéité des deux décors? Il y aurait alors deux eventualites: une pièce. non peinte jusquelà, aurait été décorée en même temps que l'on rénovait le décor de la pièce voisine; ou bien, a la suite de circonstances que nous ignorons, il y a eu réfection tolale de l'enduit dans une pièce, et simple superposition lans l'autre. Si les deux pièces chauffées n’étaient séparées que par des cloisons mobiles, on peut penser que le décor était en continuité C"est done au lepidarium qu’il faurdrait, de manière très hypothétique, altribure ce groupre.

\section{(iROUPE III.}

Couche de préparalion (fig. I d) : rose, assez compacte, de $2: 3 \mathrm{~mm}$ d'epaisseur, elle est composée de sable, de gros graviers et de tuileau, liés à la chaux. L'aspect du revers varie : on y lit parfois l'empreinte de moellons (hauts de 10 a $12 \mathrm{~cm}$ ); sur d'aut res fragments, il reste accroché un mélange terreux, beige, où se distinguent des nodules de chatux, des graviers dispersés el de la paille: couche de transition : de couleur heige, elle est peu homogine les nodules de chaux restent hien

11 X. Davey, The Conservalion of Romano-Brilish Painted Plaster, dans Brilannia, 3, 197-2, p. 251-26x, aver: reference a J. S. Wacher, Roman Brilain in 19:58, dans Journal of Roman Siludies, 19, 1959, 1. $113-115$.

12 Ouvage cite, t. II, en particulier pl. CXXV.

13 Inedit. (es peintures provenant de la rue aux ormes nous ont ite confiees par l). Joly.

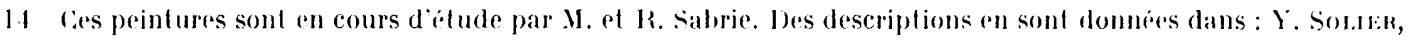

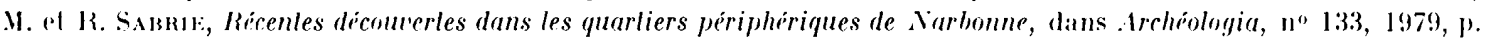

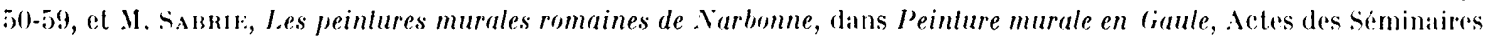

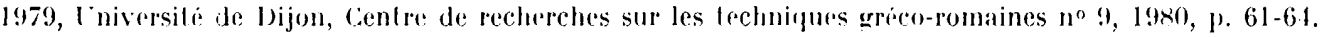




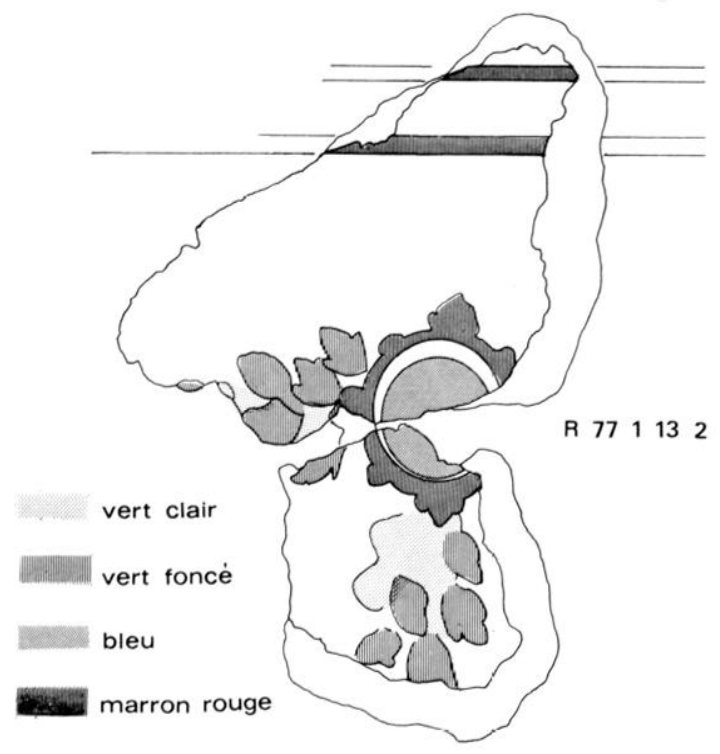

distincts): tris chargée en sable, avec de rares éléments de tuileau :- - la couche superlicielle, en chaux presque pure. a environ $1 \mathrm{~mm}$ d'ppaisseur. Lille est en tris mauvais elal de ronservation.

Plusieurs fragments, malheureusement pelits et sans assemblages possibles, présenlent des petits feuillages verts sur fond blanc R 77 I 1:3. Sur l'un d'eux apparaît une guirlande courbe $R 7 \%$ I 13-25).

sur fond blanc également, un carré à cotés roncaves bleu est rattachó par pointe a une double ligne jaune, courbe. L'empreinte des moellons du revers donne le sens de lecture du motif : la verticale passe par la diagonale de l'angle du carré (R 77 I 1:3 23). Plusieurs fragments a lignes courbes bleues appartiennent au mème ensemble.

Jeux tiges vertes, feuillues, partent per-

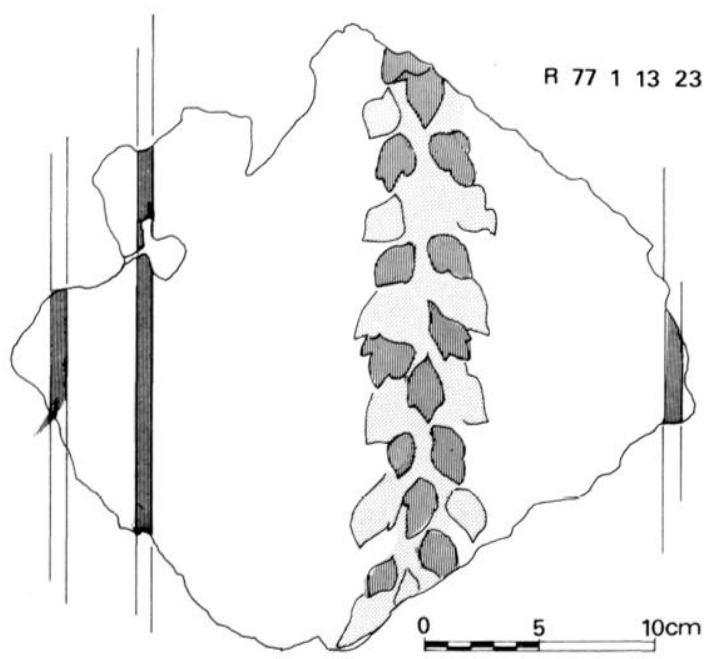
pendiculairement d'un petil disque (diam. : $18 \mathrm{~mm}$ ) bleu cerné de rouge (fig. 10). De part et d'autre de ces liges, deux doubles filets marron délimitent une bande large de $25 \mathrm{~cm}$. Cet ensemble - tiges feuillues et filets marron - constitue sans doute l'élément décoratif essentiel du compartimentage de la paroi.

La présence de disques et de guirlandes, liée à celle de carrés curvilignes rt/ou de cereles, nous avait d'abord laissé pressentir un décor de plafond. Mais l'existence des empreintes de moellons au dos d'un fragment dément cette hypotheses. Il s'agit donc bien d'un décor mural. Le mélange terreux adhérant incore sur

d'autres revers peut itre un restige de torchis. La construction des murs dont ils proviennent serait alors assez disparate : rangées de moellons à la hase, par exemple, et torchis alt-dessus ? Ou alors, ce serait une couche préliminaire, posée par endroits seulement. Ce matériau est cependant assey inhabituel dans un édifier thermal : cet ensemble pourrait plutòt provenir d'une piece annexe que des bains proprement dits. Le motif le plus reconnaissable, celui des guirlandes at des disques, est bien connu. Son utilisation la plus fréquente est un réseau régulier de petits compartiments délimités par les guirlandes, marqué aux intersections par les cercles colorés en rouge ou en bleu, caractéristique d'un décor de plafond, d'où notre premiere hẹpothese.

Vais si le principe ne est pas rare dans l'inspiration et les coloris, pen dexemples sont vraiment proches du nòtre. Caest en suisse que, gràce à une série de decors de ce type nous 
voyons le micux les variantes possibles. sur les fragments isoles provenant de Augst ${ }^{15}$ sont peints des disques beus ou rouges, auxquels l'adjonetion d'une couronne de pétales donne laspeet d'un fleuron schématique. Cäest bien le mème traitement du cerele décoratif, mais leur contexte n'est malheurensement pas connu. A Wagen ${ }^{16}$. il reste des témoins d'un réseau de gruirlandes rigides et de cercles dont nous ignorons la dimension. I a restitution du "plafond d'une villa" a . Mllaz"17 donne un ensemble plus coliérent : un quarlrillage de guirlandes droiles. arliculées sur dess disques, délimite des aissons que remplissent dess

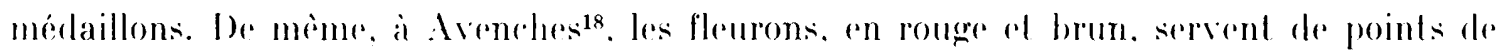
départ a des guirlandes, esquissés par des sépies de trois fenilles vertes, séparand des medaillons faits de deux cereles concentriques en rouge al jaune. A Ribemont, le lien n'est

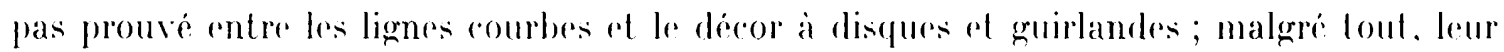
rapprochement est probable. et les comparaisons précédentes ne sont pas à négliger. Il est regrettable que, pour la plupart des vestiges que nous venons de mentionner, of qui provirnnent de fouilles anciennes. les datations soient extremement floues, ou étallies par

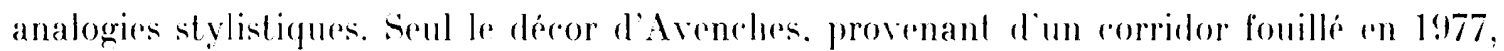
peut etre situé au délunt du nire sièrle.

Mrentionnons encore. en Cirandr-Bretagne. un plafond de Leicester oil less quirlandes vertes orthogonales, se rencontrant toujours sur les disques. se rompliquent de motifs végétaux à quatre lobes, qui orecupent la plus grande partie de la surfacere.

Il arrive exeeptionnellement quin tel systeme soit arlapte a une surface pariétale. Sous connaissons quelques exemples en Campanie de réseaux végétaux couvrant des

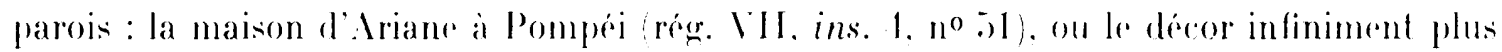
rafliné, aver intégration de mérlaillons et de figures. de la villa de larano à stabies.

Pourtant, a Ribemont, la rarete des disques par rapport a la longueur de guirlande ronservé tendrait a exclure l'hypothese d'un reoisonnement aussi serré. Nous chereherons done plutot des encadrements de panneaux qui, bien que resermblant au découpage de la surface précédemment évoqué, présentent une trame plus large.

la fouille de la villa des Boheix-Cujasseix ${ }^{20}$, en Limousin, a fourni une peinture bien

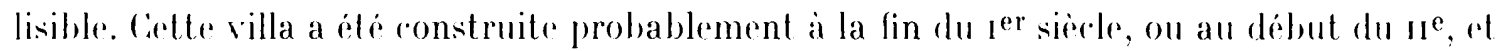
lon perut penser que le décor intérieur. sur lequel on ne lécèle aucun remaniement, lui est contemporain. Sur un fond blance des petits panneaux marrons ou verts sont encarlrés de guirlandes accrochés par des noeuds schématiques aux disques qui marquent les angles (fig. 11). Jans le cas d'un panneau marron, la guirlande est verte, le disque rouge ; dans le cas d'un panneau noir, la gruirlande est bleue et rouge, et le disque noir. (On notera l'intérêt des handes curvilignes voisines de la gruilande; a Ribemont, le lien direct entre les deux

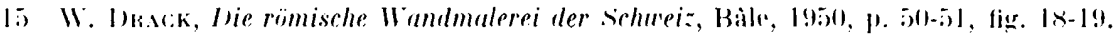

16 liem, p. 117 , figr. 120 .

17 Item, 1. 150, list. 16:3.

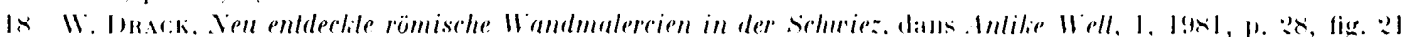

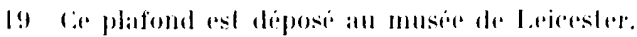

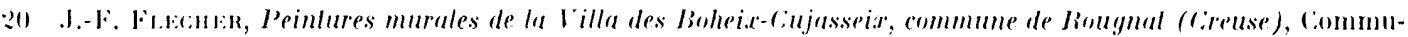

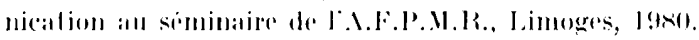




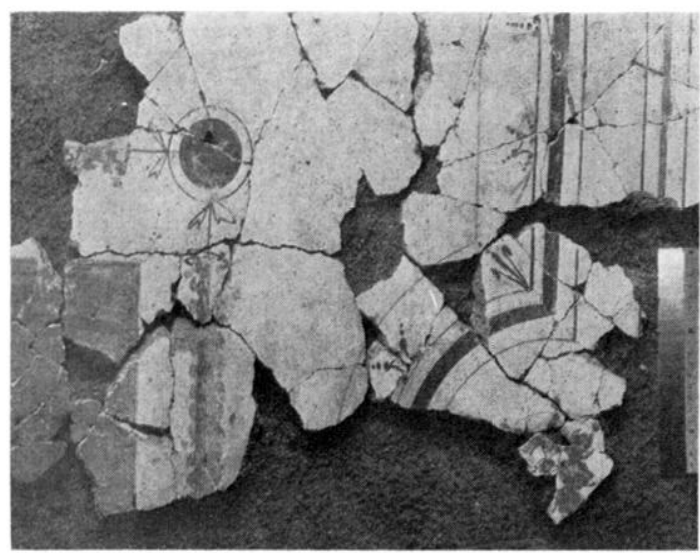

II Villa des Boheix-dajassejx, encadrement de guirlandes aver disques aux angles.

motifs nees pas trouvé : mais leur presenece dans le meme ensemble permet ce rapprochement supplémentaire. Précisons encore qu un encadrement de fenetre et des empreintes de moellons au revers promient lien que les enduits de Cujasseix proviennent des murs et non du plafond.

Ce molif encore se retrourera plus fréquemment, par la suite. dans laart musival, et la guirlande rigide séparant les différents panneaux. articulée sur des petits cercles, est une décoration fréquente, mais sur des pavements très tardifs: une mosä̈que trouvée à sens présente un encadrement similaire, ainsi que quelyues mosä̈ques africaines (maison de la Chasse à Utique en particulier). Bien entendu les décalages géographiques et chronologiques interdisent toutes comparaisons directes ; très certainement des jalons intermédiaires nous manquent, mais il apparait nettement, comme pour le groupe I, que le répertoire utilisé n'est pas spécifique a la peinture, et se trowre aussi bien sur les pavements que sur les parois.

Sous sommes donc en présence d'éléments décorés qui, bien qu assez paurers, ont l'avantage d'entrer dans des séries analogues, et de nous permettre l'avoir un échantillonnage de quelques types de décors utilisés au $\mathrm{II}^{\mathrm{e}}$ siècle. L'empreinte des moellons ou des legulae au revers des mortiers at teste la contemporanéité de la construction ct du revêtement peint. On ne peut. en effet. penser que les murs soient restés à nu quelque temps : l'enduit dans les salles thermales joue un ròle au moins aussi fonctionnel que décoratif, et est indispensable à l'isolation des piècess exposées à la chaleur et à l'humidilé. En cas de rénovation du décor peint, un nouvel enduit est simplement superposé à l'ancien. of nous en avons "ffectivement un témoin flagrant.

L'hypothèse la plus satisfaisante est done d'attribuer ces peintures à la mème date que celle de la construction (époque trajane) ; puis une réfection partielle au moment d'une série de rénovations situer a l'époque de Vare-Aurele. ()n ne saurait loul efois exelure qüil y ail, en, a celte derniere période. un remaniement de l'onsemble de lédifice ; dans ree cas, maçonnerie el peinture seraient a situer vers les annés 1600-170, el la date du deruxieme etat du décor impossible à préciser. 
Nous voyons que l'ètude d'un groupe d'enduits aussi fragmentaire ne permet certes pas d'aboutir à une restitution d'ensemble des parois, ni mème a une vision précise du système ornemental employé. Mais l'on constate que ces vestiges de peinture, si dispersés soient-ils, ne sont pas à négliger : l'analyse des différents enduits peut donner des indiees complémentaires de ceux de la fouille; la prise en considération de chaque élément décoratif conservé fournit une idée du répertoire utilisé, et débouche éventuellement sur des comparaisons possibles avere des découvertes analogues.

Claudine AlLAC;

(C.N.IS.S.)

N.B. Origine des documents : A. Barbel, fig. 2a et 6 ; Th. Adam, fig. 2h, at 7 ; A. Eristor; fig. 8 ; J.-F. Flecher, fig. 11. Toutes les autres illustrations sont de l'auteur. 